\title{
An auto-realignment method in quasi-Monte Carlo for pricing financial derivatives with jump structures
}

\author{
Chengfeng Weng ${ }^{\mathrm{a}}$, Xiaoqun Wang ${ }^{\mathrm{a}}$, Zhijian He $\mathrm{He}^{\mathrm{b}, *}$ \\ ${ }^{a}$ Department of Mathematical Sciences, Tsinghua University, Beijing 100084, China \\ ${ }^{b}$ Lingnan (University) College, Sun Yat-sen University, Guangzhou 510275, China
}

\begin{abstract}
Discontinuities are common in the pricing of financial derivatives and have a tremendous impact on the accuracy of quasi-Monte Carlo (QMC) method. While if the discontinuities are parallel to the axes, good efficiency of the QMC method can still be expected. By realigning the discontinuities to be axes-parallel, Wang and Tan (2013) succeeded in recovering the high efficiency of the QMC method for a special class of functions. Motivated by this work, we propose an auto-realignment method to deal with more general discontinuous functions. The $k$-means clustering algorithm, a classical algorithm of machine learning, is used to select the most representative normal vectors of the discontinuity surface. By applying this new method, the discontinuities of the resulting function are realigned to be friendly for the QMC method. Numerical experiments demonstrate that the proposed method significantly improves the performance of the QMC method.
\end{abstract}

Keywords:

Pricing, QMC, OT method, QR decompositon, auto-realignment method 2010 MSC: 65C05, 65D30, 91G20, 91G60

\section{Introduction}

Monte Carlo (MC) method and quasi-Monte Carlo (QMC) method have been successfully used to a wide range of finance problems, such as the pricing and hedging of complex financial derivatives (see Glasserman, 2004; Jin

\footnotetext{
${ }^{*}$ Corresponding author. Tel.: +86 13450361878.

Email addresses: wengcf130mails.tsinghua.edu.cn (Chengfeng Weng), xwang@math.tsinghua.edu.cn (Xiaoqun Wang), hezhijian87@gmail.com (Zhijian He)
} 
et al., 2013). By suitable transformations, these problems can often be represented as the integral of a real-valued function $H$ over the $d$-dimensional unit cube $I(H)=\int_{[0,1]^{d}} H(\boldsymbol{u}) d \boldsymbol{u}$. The QMC method approximates the integral by $Q_{N}(H)=\frac{1}{N} \sum_{i=1}^{N} H\left(\boldsymbol{u}_{i}\right)$, where the quadrature points $\boldsymbol{u}_{1}, \ldots, \boldsymbol{u}_{N}$ are deterministically chosen low discrepancy points from $[0,1]^{d}$ instead of random ones in MC sampling. The low discrepancy points are relatively uniform so that it is common for QMC to produce much more accurate answers than MC does. The justification is through the well-known Koksma-Hlawka inequality, which claims that the QMC integration error is of $O\left(N^{-1}(\log N)^{d}\right)$ for functions with finite variation in the sense of Hardy and Krause (see Niederreiter, 1992).

The advantage of the QMC method for high-dimensional finance problems was shown in many empirical studies (see Joy et al., 1996; Paskov and Traub, 1995). The concept of effective dimension offers a possible explanation for the success of QMC method for high-dimensional problems (see Wang and Fang, 2003). It is widely believed that functions of small effective dimension are easier to integrate by the QMC method. To this end, some path generation methods (PGMs) are proposed to reduce the effective dimension, such as the Brownian bridge (BB) (Caflisch et al., 1997; Moskowitz and Caflisch, 1996), the principal component analysis (PCA) (Acworth et al., 1998) and the linear transformation (LT) method (Imai and Tan, 2006). However, they do not consider the discontinuities of underlying functions.

In practice, a broad class of finance problems involves discontinuities. If the boundaries of discontinuity are not parallel to the axes, these discontinuities may be detrimental to the QMC method. The efficiency of the QMC method could be recovered if the discontinuities are realigned to be axes-parallel. Wang and Tan (2013) proposed the orthogonal transformation (OT) method for a particular class of discontinuous functions and suggested to realign discontinuities onto axes-parallel hyperplanes. Subsequently, He and Wang (2014) developed a PGM called the $\mathrm{QR}^{1}$ method to deal with multiple discontinuity structures. The QR method makes the surfaces of discontinuities parallel to as many axes as possible. Both the OT and QR methods improve the efficiency of the QMC method significantly for many problems with discontinuous functions arising from finance. Wang (2016)

\footnotetext{
${ }^{1}$ The term "QR" is from the QR decomposition in linear algebra (see Golub and van Loan, 2013).
} 
proposed a procedure to handle high dimensionality and discontinuities concurrently. But for payoffs involving complex discontinuity structures, the OT and QR methods cannot be applied directly.

In this paper, we propose a new PGM called the auto-realignment method to deal with payoffs with complex jump structures. Our approach obtains the information of the discontinuity surface from a set of low discrepancy points. We use the $k$-means clustering algorithm to automatically select several representative hyperplanes to approximate the discontinuity surface. These hyperplanes are characterized by their corresponding normal vectors. The basic idea of the auto-realignment method is intuitive: The more points distribute around the discontinuity surface with a certain normal vector, the more important is this normal vector. We could apply the QR method to realign these hyperplanes such that they are parallel to as many axes as possible. The new method can make the discontinuities of the payoffs more QMC-friendly. The auto-realignment method merits several advantages: It selects representative normal vectors and quantifies the importance of chosen vectors automatically without tedious recognition. Moreover, the performance of the auto-realignment method is more effective and robust than other PGMs, as confirmed by the numerical experiments.

The rest of the paper is organized as follows. The OT method is reviewed in Section 2. The proposed auto-realignment method is formally presented in Section 3. Section 4 performs some numerical experiments for pricing exotic options. Section 5 concludes this paper.

\section{Problem formulation and the OT method revisited}

\subsection{Problem formulation}

Suppose that $S(t)$ is the price of the underlying asset at time $t$. We assume that under the risk-neutral measure the underlying asset follows a geometric Brownian motion (BM):

$$
d S(t)=r S(t) d t+\sigma S(t) d B(t)
$$

where $r$ represents the risk-free interest rate, $\sigma$ represents the volatility, and $B(t)$ is the standard BM. The explicit solution of $(1)$ is

$$
S(t)=S(0) \exp \left\{\left(r-\sigma^{2} / 2\right) t+\sigma B(t)\right\}
$$


Let $K$ and $T$ denote the strike price and the maturity of the option, respectively. Let $\boldsymbol{S}:=\left(S\left(t_{1}\right), S\left(t_{2}\right), \ldots, S\left(t_{d}\right)\right)^{T}$ represent the $d$-dimensional vector of asset prices, where $t_{j}=j \Delta t, \Delta t=T / d$, and

$$
S\left(t_{j}\right)=S(0) \exp \left\{\left(r-\sigma^{2} / 2\right) t_{j}+\sigma B\left(t_{j}\right)\right\}, \quad j=1, \ldots, d .
$$

Let $\boldsymbol{x}=\left(x_{1}, \ldots, x_{d}\right)^{T}:=\left(B\left(t_{1}\right), \ldots, B\left(t_{d}\right)\right)^{T}$, then $\boldsymbol{x} \sim N(\mathbf{0}, \boldsymbol{C})$, where $C$ is the covariance matrix with entries $C_{i j}=\Delta t \min (i, j), i, j=1, \ldots, d$. Suppose that the discounted payoff function of a path-dependent option is given by $H(\boldsymbol{x})$. The price of the option can be expressed as $\mathbb{E}[H(\boldsymbol{x})]$, where $\mathbb{E}[\cdot]$ is the expectation under the risk-neutral measure.

Let $\boldsymbol{A}$ be any decomposition of $\boldsymbol{C}$ such that $\boldsymbol{A} \boldsymbol{A}^{T}=\boldsymbol{C}$ and $\boldsymbol{z}$ be a $d$ dimensional vector of independent standard normal random variables, i.e., $\boldsymbol{z} \sim N\left(\mathbf{0}, \boldsymbol{I}_{d}\right)$, then we have $\boldsymbol{A} \boldsymbol{z} \sim N(\mathbf{0}, \boldsymbol{C})$. The matrix $\boldsymbol{A}$ is called the generating matrix of $\boldsymbol{x} \sim N(\mathbf{0}, \boldsymbol{C})$. From the view of expectation, the price can be written as

$$
V(H):=\mathbb{E}[H(\boldsymbol{A z})]=\mathbb{E}\left[H\left(\boldsymbol{A} \Phi^{-1}(\boldsymbol{u})\right)\right],
$$

where $\boldsymbol{u}$ is uniformly distributed on $[0,1]^{d}$ and $\Phi$ is the cumulative distribution function of standard normal. It can be approximated by

$$
Q_{N}(H, \mathcal{P}, \boldsymbol{A})=\frac{1}{N} \sum_{i=1}^{N} H\left(\boldsymbol{A} \Phi^{-1}\left(\boldsymbol{u}_{i}\right)\right),
$$

where $\mathcal{P}=\left\{\boldsymbol{u}_{i}, i=1, \ldots, N\right\}$ is a set of low discrepancy points in $[0,1]^{d}$. It should be stressed that the choice of the generating matrix $\boldsymbol{A}$ is not unique. The accuracy of the approximation $Q_{N}(H, \mathcal{P}, \boldsymbol{A})$ may depend heavily on the choice of $\boldsymbol{A}$. The generating matrix of the standard (STD) construction of the $\mathrm{BM}$ is given by

$$
\boldsymbol{A}^{\mathrm{STD}}:=\sqrt{\Delta t}\left[\begin{array}{cccc}
1 & 0 & \cdots & 0 \\
1 & 1 & \cdots & 0 \\
\vdots & \vdots & \ddots & \vdots \\
1 & 1 & \cdots & 1
\end{array}\right]
$$

which is actually the Cholesky decomposition of the covariance matrix $\boldsymbol{C}$. Since the STD, BB and PCA constructions for the BM do not take the payoff functions into account, they may have bad performance for payoff functions with jump structures. In the next subsection, we review the OT method that deals with simple discontinuities. 


\subsection{The OT method revisited}

The discontinuities which are axes-parallel are considered as QMC-friendly since high efficiency can still be expected in QMC. Wang and Tan (2013) proposed the OT method that transforms the discontinuities to be QMCfriendly. The OT method targets the following special class of discontinuous functions

$$
H(\boldsymbol{x})=\mathbf{1}_{\left\{h\left(\boldsymbol{q}^{T} \boldsymbol{x}\right)>0\right\}}(\boldsymbol{x}),
$$

where $\boldsymbol{q}=\left(q_{1}, \ldots, q_{d}\right)^{T}$ is a nonzero vector of constants and $h(\cdot)$ is a strictly increasing function defined on $\mathbb{R}$.

Let $\boldsymbol{A}_{0}$ be an arbitrary initial generating matrix satisfying $\boldsymbol{A}_{0} \boldsymbol{A}_{0}^{T}=\boldsymbol{C}$ and $\boldsymbol{p}^{T}:=\left(p_{1}, \ldots, p_{d}\right)=\boldsymbol{q}^{T} \boldsymbol{A}_{0}$. The payoff function (4) is equivalent to

$$
H(\boldsymbol{z})=\mathbf{1}_{\left\{h\left(\boldsymbol{q}^{T} \boldsymbol{A}_{0} \boldsymbol{z}\right)>0\right\}}(\boldsymbol{z})=\mathbf{1}_{\left\{h\left(\boldsymbol{p}^{T} \boldsymbol{z}\right)>0\right\}}(\boldsymbol{z})=\mathbf{1}_{\left\{\boldsymbol{p}^{T} \boldsymbol{z}>h^{-1}(0)\right\}}(\boldsymbol{z}),
$$

where $\boldsymbol{z}=\left(z_{1}, \ldots, z_{d}\right)^{T} \sim N\left(\mathbf{0}, \boldsymbol{I}_{d}\right)$. By using the inverse transformation $\boldsymbol{z}=\Phi^{-1}(\boldsymbol{u})$, the inequality in (5) can be written as

$$
p_{1} \Phi^{-1}\left(u_{1}\right)+p_{2} \Phi^{-1}\left(u_{2}\right)+\cdots+p_{d} \Phi^{-1}\left(u_{d}\right)>h^{-1}(0) .
$$

Obviously, the discontinuity boundary may not be parallel to any coordinate axis.

The OT method offers a precise specification of an orthogonal matrix $\boldsymbol{U}$ by setting the first column of $\boldsymbol{U}$ as $\boldsymbol{U}_{\cdot 1}=\frac{1}{D} \boldsymbol{A}_{0}^{T} \boldsymbol{q}$, where $D=\sqrt{\boldsymbol{q}^{T} \boldsymbol{C q}}$ and the remaining columns of $\boldsymbol{U}$ are arbitrary as long as they satisfy the orthogonality conditions. The OT method takes the generating matrix as $\boldsymbol{A}^{\mathrm{OT}}=\boldsymbol{A}_{0} \boldsymbol{U}$. By the transformation $\boldsymbol{x}=\boldsymbol{A}^{\mathrm{OT}} \boldsymbol{z}$, the expectation $\mathbb{E}[H(\boldsymbol{x})]$ can be represented as

$$
\mathbb{E}\left[\mathbf{1}_{\left\{h\left(\boldsymbol{q}^{T} \boldsymbol{A}_{0} \boldsymbol{U} \boldsymbol{z}\right)>0\right\}}(\boldsymbol{z})\right]=\mathbb{E}\left[\mathbf{1}_{\left\{h\left(D z_{1}\right)>0\right\}}(\boldsymbol{z})\right] .
$$

The indicator function (4) is transformed to

$$
\mathbf{1}_{\left\{h\left(D z_{1}\right)>0\right\}}(\boldsymbol{z})=\mathbf{1}_{\left\{z_{1}>\frac{1}{D} h^{-1}(0)\right\}}(\boldsymbol{z}) .
$$

By the transformation $\boldsymbol{z}=\Phi^{-1}(\boldsymbol{u})$, the inequality in (6) can be further written as

$$
u_{1}>\Phi\left(D^{-1} h^{-1}(0)\right) \text {. }
$$

Note that the discontinuity occurs at $u_{1}=\Phi\left(D^{-1} h^{-1}(0)\right)$. Hence the discontinuity surface of the payoff function is realigned to be QMC-friendly. 
The OT method mainly focuses on indicator functions with respect to one linear structure $\boldsymbol{q}^{T} \boldsymbol{x}$. But for payoffs involving complex discontinuities, it is difficult to figure out the right generating matrix $\boldsymbol{A}$ such that the discontinuities are realigned to be QMC-friendly.

\section{The auto-realignment method}

Since the nature of discontinuities of the underlying functions has a tremendous impact on the accuracy of the QMC method, the success of the OT method motivates us to develop a general PGM for payoff functions involving complex discontinuity structures.

\subsection{The fundamentals}

Suppose that $\boldsymbol{A}_{0}$ is an initial generating matrix satisfying $\boldsymbol{A}_{0} \boldsymbol{A}_{0}^{T}=\boldsymbol{C}$, then $\boldsymbol{A} \boldsymbol{A}^{T}=\boldsymbol{C}$ if and only if $\boldsymbol{A}$ can be expressed as $\boldsymbol{A}=\boldsymbol{A}_{0} \boldsymbol{U}$ for some orthogonal matrix $\boldsymbol{U}$. Assume that the payoff function is written as

$$
h(\boldsymbol{z})=H\left(\boldsymbol{A}_{0} \boldsymbol{z}\right)=h_{0}(\boldsymbol{z}) \mathbf{1}_{\{f(\boldsymbol{z})>0\}}(\boldsymbol{z}), \boldsymbol{z} \sim N\left(\mathbf{0}, \boldsymbol{I}_{d}\right),
$$

where $h_{0}$ and $f$ are almost everywhere differentiable in $\mathbb{R}^{d}$. Note that $f(\boldsymbol{z})>$ 0 is the condition of exercising and $h_{0}(\boldsymbol{z})$ is the payoff amount when the security is exercised. The discontinuity surface of $h(\boldsymbol{z})$ is

$$
\Sigma:=\{\boldsymbol{z} \mid f(\boldsymbol{z})=0\} .
$$

Let $\varepsilon_{1}, \ldots, \varepsilon_{r}$ be $r$ arbitrary fixed points on the discontinuity surface $\Sigma$ and let $\mathrm{H}_{1}, \ldots, \mathrm{H}_{r}$ be the corresponding local discontinuity hyperplane in the neighborhood of $\varepsilon_{1}, \ldots, \varepsilon_{r}$, defined by

$$
\mathrm{H}_{i}:=\left\{\boldsymbol{z} \mid \nabla f\left(\boldsymbol{\varepsilon}_{i}\right)^{T}\left(\boldsymbol{z}-\boldsymbol{\varepsilon}_{i}\right)=0\right\}, i=1, \ldots, r,
$$

where $\nabla f\left(\boldsymbol{\varepsilon}_{i}\right)$ is the gradient vector of $f(\boldsymbol{z})$ evaluated at $\boldsymbol{\varepsilon}_{i}$. The normal vector of the local discontinuity hyperplane $\mathrm{H}_{i}$ is

$$
\boldsymbol{l}_{i}:=\frac{\nabla f\left(\varepsilon_{i}\right)}{\left\|\nabla f\left(\varepsilon_{i}\right)\right\|}, i=1, \ldots, r .
$$

For an orthogonal matrix $\boldsymbol{U}$ satisfying $\boldsymbol{U} \boldsymbol{U}^{T}=\boldsymbol{I}$, let $\tilde{\boldsymbol{z}}=\boldsymbol{U}^{T} \boldsymbol{z}$. Then $\boldsymbol{z}=\boldsymbol{U} \tilde{\boldsymbol{z}}$ and the payoff function in (7) can be written as

$$
h(\boldsymbol{z})=h(\boldsymbol{U} \tilde{\boldsymbol{z}})=h_{0}(\boldsymbol{U} \tilde{\boldsymbol{z}}) \mathbf{1}_{\{f(\boldsymbol{U} \tilde{\boldsymbol{z}})>0\}}(\tilde{\boldsymbol{z}}) .
$$


Let $\tilde{h}(\tilde{\boldsymbol{z}})=h(\boldsymbol{U} \tilde{\boldsymbol{z}})$ and $\tilde{f}(\tilde{\boldsymbol{z}})=f(\boldsymbol{U} \tilde{\boldsymbol{z}})$. We have $\mathbb{E}[h(\boldsymbol{z})]=\mathbb{E}[\tilde{h}(\tilde{\boldsymbol{z}})]$. The discontinuity surface of $\tilde{h}(\tilde{\boldsymbol{z}})$ is $\tilde{\Sigma}=\{\tilde{\boldsymbol{z}} \mid \tilde{f}(\tilde{\boldsymbol{z}})=0\}$. Note that there exists a one-to-one correspondence between $\Sigma$ and $\tilde{\Sigma}$ under the mapping $\phi$ :

$$
\phi: \boldsymbol{z} \mapsto \tilde{\boldsymbol{z}}=\boldsymbol{U}^{T} \boldsymbol{z} .
$$

The points $\varepsilon_{1}, \ldots, \boldsymbol{\varepsilon}_{r}$ correspond to the points $\tilde{\boldsymbol{\varepsilon}}_{1}, \ldots, \tilde{\boldsymbol{\varepsilon}}_{r}$ on the new discontinuity surface. Denote $\tilde{\mathrm{H}}_{1}, \ldots, \tilde{\mathrm{H}}_{r}$ as the new local discontinuity hyperplanes in the neighborhoods of $\tilde{\varepsilon}_{1}, \ldots, \tilde{\varepsilon}_{r}$, defined by

$$
\tilde{\mathrm{H}}_{i}:=\left\{\tilde{\boldsymbol{z}} \mid \nabla \tilde{f}\left(\tilde{\boldsymbol{\varepsilon}}_{i}\right)^{T}\left(\tilde{\boldsymbol{z}}-\tilde{\boldsymbol{\varepsilon}}_{i}\right)=0\right\}, i=1, \ldots, r .
$$

The normal vectors of the new local discontinuity hyperplane $\tilde{\mathrm{H}}_{1}, \ldots, \tilde{\mathrm{H}}_{r}$ can be denoted as $\tilde{\boldsymbol{l}}_{1}, \ldots, \tilde{\boldsymbol{l}}_{r}$, where

$$
\tilde{\boldsymbol{l}}_{i}:=\frac{\nabla \tilde{f}\left(\tilde{\varepsilon}_{i}\right)}{\left\|\nabla \tilde{f}\left(\tilde{\varepsilon}_{i}\right)\right\|}, i=1, \ldots, r .
$$

Theorem 1. Let $h(\boldsymbol{z})$ be the payoff function given in (7) and $\Sigma$ be the discontinuity surface given in (8). Let $\varepsilon_{1}, \ldots, \boldsymbol{\varepsilon}_{r}$ be $r$ arbitrary fixed points on the discontinuity surface $\Sigma$. The normal vectors $\boldsymbol{l}_{1}, \ldots, \boldsymbol{l}_{r}$ on these points of the discontinuity surface are given by (10). Let $\boldsymbol{L}=\left(\boldsymbol{l}_{1}, \ldots, \boldsymbol{l}_{r}\right) \in \mathbb{R}^{d \times r}$ and assume that $\operatorname{Rank}(\boldsymbol{L})=r<d$. Let $\boldsymbol{Q}$ be the resulting orthogonal matrix from the $Q R$ decomposition $\boldsymbol{L}=\boldsymbol{Q} \boldsymbol{R}$, where $\boldsymbol{R} \in \mathbb{R}^{d \times r}$ is an upper triangular matrix. Then by setting $\boldsymbol{U}=\boldsymbol{Q}$, the new local discontinuity hyperplane $\tilde{H}_{i}$ given in (11) is aligned parallel to the latter $d-i$ axes.

Proof. Note that $\tilde{f}(\tilde{\boldsymbol{z}})=f(\boldsymbol{U} \tilde{\boldsymbol{z}})$. By the chain rule of differentiation, we have

$$
\left.\frac{\partial \tilde{f}}{\partial \tilde{z}_{i}}\right|_{\tilde{z}=\tilde{\varepsilon}_{1}}=\nabla f\left(\boldsymbol{\varepsilon}_{1}\right)^{T} \boldsymbol{U}_{\cdot i}, i=1, \ldots, d,
$$

where $\boldsymbol{U}_{. i}$ is the $i$ th column of orthogonal matrix $\boldsymbol{U}$. Note that $\boldsymbol{U} \boldsymbol{U}^{T}=\boldsymbol{I}$ and $\boldsymbol{R} \in \mathbb{R}^{d \times r}$ is an upper triangular matrix. The normal vector $\tilde{\boldsymbol{l}}_{1}$ of the local discontinuity hyperplane $\tilde{\mathrm{H}}_{1}$ is

$$
\tilde{\boldsymbol{l}}_{1}=c_{1}^{\prime} \boldsymbol{U}^{T} \nabla f\left(\boldsymbol{\varepsilon}_{1}\right)=c_{1} \boldsymbol{U}^{T} \boldsymbol{l}_{1}=c_{1} \boldsymbol{U}^{T} \boldsymbol{U} \boldsymbol{R}_{1}=c_{1}\left(R_{11}, 0, \ldots, 0\right)^{T},
$$

where $\boldsymbol{R}_{i}$ is the $i$ th column of the matrix $\boldsymbol{R}$ and $c_{1}^{\prime}, c_{1}$ are appropriate coefficients such that $\left\|\tilde{\boldsymbol{l}}_{1}\right\|=1$. This implies that $\tilde{\mathrm{H}}_{1}$ is parallel to $d-1$ 
axes. Next we consider the normal vector $\tilde{\boldsymbol{l}}_{2}$ of the new local discontinuity hyperplane $\tilde{\mathrm{H}}_{2}$. Similarly, we have

$$
\tilde{\boldsymbol{l}}_{2}=c_{2}^{\prime} \boldsymbol{U}^{T} \nabla f\left(\boldsymbol{\varepsilon}_{2}\right)=c_{2} \boldsymbol{U}^{T} \boldsymbol{l}_{2}=c_{2} \boldsymbol{U}^{T} \boldsymbol{U} \boldsymbol{R}_{\cdot 2}=c_{2}\left(R_{12}, R_{22}, 0, \ldots, 0\right)^{T},
$$

where $c_{2}^{\prime}$ and $c_{2}$ are appropriate coefficients such that $\left\|\tilde{\boldsymbol{l}}_{2}\right\|=1$. This implies that $\tilde{\mathrm{H}}_{2}$ is parallel to the later $d-2$ axes. The following steps are similar to the previous deduction.

\subsection{Finding representative normal vectors of the discontinuity surface}

How to find the representative normal vectors on the discontinuity surface? Our strategy is to obtain a whole picture of normal vectors on the discontinuity surface from a number of sample points. Then we use $k$-means clustering algorithm to automatically select several most representative normal vectors on the discontinuity surface. The details are stated as Steps (1)(3) below.

Step (1) Sample $n$ points $\varepsilon_{1}, \ldots, \varepsilon_{n}$ around the discontinuity surface $\Sigma$ and calculate their corresponding normal vectors $\boldsymbol{v}_{1}, \ldots, \boldsymbol{v}_{n}$ of the discontinuity surface.

Theoretically it is difficult to choose the required points exactly on $\Sigma$. Thus an area with positive probability $\{\boldsymbol{z}:|f(\boldsymbol{z})|<\eta\}$ is used as a substitute of the discontinuity surface, where $\eta$ is a small number. Generate $\varepsilon_{1}, \ldots, \boldsymbol{\varepsilon}_{N}$ from low discrepancy points $\boldsymbol{u}_{1}, \ldots, \boldsymbol{u}_{N} \in[0,1]^{d}$ by the transformation $\varepsilon=\Phi^{-1}(\boldsymbol{u})$. These points $\varepsilon_{1}, \ldots, \varepsilon_{N} \in \mathbb{R}^{d}$ are considered around the discontinuity surface when the condition $\left|f\left(\varepsilon_{i}\right)\right|<\eta$ holds. In our experiment, $N=2^{14}$ and $\eta$ is the 1 st percentile of $N$ observations $\left|f\left(\varepsilon_{1}\right)\right|, \ldots,\left|f\left(\varepsilon_{N}\right)\right|$. For simplicity, we assume that the first $n$ points $\varepsilon_{1}, \ldots, \varepsilon_{n}$ are selected according to the condition $\left|f\left(\varepsilon_{i}\right)\right|<\eta$. Thus we are able to obtain a group of normal vectors $\boldsymbol{v}_{1}, \ldots, \boldsymbol{v}_{n}$ of the discontinuity surface on these selected points.

Step (2) Apply the $k$-means clustering algorithm proposed by Hartigan and Wong (1979) to partition $\boldsymbol{v}_{1}, \ldots, \boldsymbol{v}_{n}$ into $r$ clusters $\mathcal{C}_{1}, \ldots, \mathcal{C}_{r}$. Choose $\boldsymbol{l}_{1}, \ldots, \boldsymbol{l}_{r}$ as the centroids of each cluster such that the within-cluster sum of distance to centroids is minimized. Choosing the number of clusters $r$ is a practical issue; see Remark 1 for details. 
The clustering algorithm is run by a cyclic process. Let $\mathcal{C}:=\left\{\boldsymbol{v}_{1}, \ldots, \boldsymbol{v}_{n}\right\}$ and denote $\boldsymbol{l}_{1}^{(i)}, \ldots, \boldsymbol{l}_{r}^{(i)}$ as the $i$ th step centroids and $\mathcal{C}_{1}^{(i)}, \ldots, \mathcal{C}_{r}^{(i)}$ as the $i$ th step clusters.

Firstly, we select $r$ vectors $\boldsymbol{l}_{1}^{(1)}, \ldots, \boldsymbol{l}_{r}^{(1)}$ randomly from $\mathcal{C}$ as the 1 st step centroids. Then $\mathcal{C}$ can be partitioned into $r$ clusters $\mathcal{C}_{1}^{(1)}, \ldots, \mathcal{C}_{r}^{(1)}$ according to the following criterion,

$$
\mathcal{C}_{i}^{(1)}:=\left\{\boldsymbol{v} \in \mathcal{C}: \rho\left(\boldsymbol{v}, \boldsymbol{l}_{i}^{(1)}\right) \leq \rho\left(\boldsymbol{v}, \boldsymbol{l}_{j}^{(1)}\right), \forall 1 \leq j \leq r\right\}
$$

where $\rho(\boldsymbol{x}, \boldsymbol{y})=1-\cos \langle\boldsymbol{x}, \boldsymbol{y}\rangle$ denotes the distance between vectors $\boldsymbol{x}$ and $\boldsymbol{y}$. The distance $\rho(\boldsymbol{x}, \boldsymbol{y})$ measures the divergence to which extent the vector $\boldsymbol{x}$ is from the vector $\boldsymbol{y}$. After $\mathcal{C}$ being partitioned into 1 st step clusters $\mathcal{C}_{1}^{(1)}, \ldots, \mathcal{C}_{r}^{(1)}$, the centroid of the $i$ th cluster is then updated by

$$
\boldsymbol{l}_{i}^{(2)}=c \sum_{\boldsymbol{v} \in \mathcal{C}_{i}^{(1)}} \boldsymbol{v}
$$

where $c$ is the normalization factor such that $\left\|\boldsymbol{l}_{i}^{(2)}\right\|=1$. Steps (12) and (13) can be described as the assignment step and the update step, respectively. Given the centroids $\boldsymbol{l}_{1}^{(2)}, \ldots, \boldsymbol{l}_{r}^{(2)}$, the group of normal vectors $\boldsymbol{v}_{1}, \ldots, \boldsymbol{v}_{n}$ can be reassigned based on the reassignment step to produce the new clusters $\mathcal{C}_{1}^{(2)}, \ldots, \mathcal{C}_{r}^{(2)}$. The algorithm proceeds by alternating between the assignment step and the update step until vectors in each cluster no longer change. In the end, we get the convergence clusters $\mathcal{C}_{1}, \ldots, \mathcal{C}_{r}$ with centroids $\boldsymbol{l}_{1}, \ldots, \boldsymbol{l}_{r}$.

Step (3) Arrange the chosen vectors $\boldsymbol{l}_{1}, \ldots, \boldsymbol{l}_{r}$ in a decreasing order $\boldsymbol{l}_{1}^{*}, \ldots, \boldsymbol{l}_{r}^{*}$ according to their importance. The weight matrix is chosen as $\boldsymbol{L}=$ $\left(\boldsymbol{l}_{1}^{*}, \ldots, \boldsymbol{l}_{r}^{*}\right) \in \mathbb{R}^{d \times r}$.

It is natural to quantify the importance of certain normal vector $\boldsymbol{l}_{i}$ by the number of vectors clustered in the set $\mathcal{C}_{i}$. The more vectors amass in $\mathcal{C}_{i}$, the more frequently random points distribute around the discontinuity surface with the normal vector $\boldsymbol{l}_{i}$, which implies that this structure is more important. Namely, the normal vector $\boldsymbol{l}_{1}^{*}$ is the centroid of the cluster which contains the most vectors, and $\boldsymbol{l}_{r}^{*}$ is the centroid of the cluster which contains the least vectors. That is why we choose the weight matrix as $\boldsymbol{L}=\left(\boldsymbol{l}_{1}^{*}, \ldots, \boldsymbol{l}_{r}^{*}\right)$, whose columns are the representative normal vectors of the discontinuity. 


\subsection{Realigning the discontinuities}

After obtaining the weight matrix $\boldsymbol{L}$, as suggested by Theorem 1, we perform a $\mathrm{QR}$ decomposition of $\boldsymbol{L}$ such that $\boldsymbol{L}=\boldsymbol{Q} \boldsymbol{R}$, where $\boldsymbol{Q}$ is an orthogonal matrix and $\boldsymbol{R}$ is an upper triangular matrix. We find the orthogonal matrix $\boldsymbol{U}$ of the auto-realignment method by setting $\boldsymbol{U}=\boldsymbol{Q}$. The resulting generating matrix is given by $\boldsymbol{A}^{\mathrm{AUTO}}=\boldsymbol{A}_{0} \boldsymbol{U}$, where $\boldsymbol{A}_{0}$ is the initial generating matrix given in (7).

We start with this section by verifying that the orthogonal matrix $\boldsymbol{U}$ indeed makes the discontinuities of the payoff function (7) more QMC-friendly. Recall that the new discontinuity surface of the payoff function is given as $\tilde{\Sigma}=\{\tilde{\boldsymbol{z}} \mid f(\boldsymbol{U} \tilde{\boldsymbol{z}})=0\}$. It is enough to verify that the normal vector on the surface $\Sigma$ is nearly parallel to many axes.

Note that the discontinuity surface $\Sigma$ is well approximated by hyperplanes with normal vectors $\boldsymbol{l}_{1}^{*}, \ldots, \boldsymbol{l}_{r}^{*}$ in Step $(2)$. For any point $\tilde{\boldsymbol{z}}=\left(\tilde{z}_{1}, \ldots, \tilde{z}_{d}\right)$ on the discontinuity surface $\tilde{\Sigma}, \tilde{f}(\tilde{\boldsymbol{z}})=0$ is equivalent to $f(\boldsymbol{z})=0$. Without loss of generality, assume that the normal vector on $z$ of the discontinuity surface belongs to the cluster with the centroid $\boldsymbol{l}_{1}^{*}$. We could reasonably assume that the normal vector of the local discontinuity hyperplane on $\boldsymbol{z}$ can be approximated by $\boldsymbol{l}_{1}^{*}$. Thus the new local discontinuity hyperplane on $\tilde{\boldsymbol{z}}$ is nearly realigned parallel to the latter $d-1$ axes according to Theorem 1 . Note that the new local discontinuity hyperplane on $\tilde{\boldsymbol{z}}$ is given by

$$
\tilde{\mathrm{H}}=\left\{\boldsymbol{z} \mid\|\nabla \tilde{f}(\tilde{\boldsymbol{z}})\|\left(z_{1}-\tilde{z}_{1}\right)=0\right\} .
$$

By the transformation $\boldsymbol{z}=\Phi^{-1}(\boldsymbol{u})$, the local hyperplane (14) can be further

transformed into $\tilde{\mathrm{H}}=\left\{\boldsymbol{u} \mid u_{1}=\Phi\left(\tilde{z}_{1}\right)\right\}$. Thus the discontinuity surface $\Sigma$ of the payoff function is transformed into a new discontinuity surface $\tilde{\Sigma}$, which is more QMC-friendly.

Remark 1. In order to apply the auto-realignment method, we need to specify the number of clusters $r$. Here we suggest practical criteria for choosing $r$. Let $\operatorname{AQR}(r), r \in \mathbb{N}$, denote the auto-realignment method which chooses $r$ clusters. Since it is inefficient to choose nearly parallel normal vectors for the weight matrix $\boldsymbol{L}$, we need to set a tolerance level $\epsilon$ to keep chosen normal vectors distinct from each other. Denote $\mathrm{AQR}^{*}$ as the auto-realignment method choosing $r^{*}$ hyperplanes to approximate the discontinuity surface, i.e., $\operatorname{AQR}^{*}:=\operatorname{AQR}\left(r^{*}\right)$, in which

$$
r^{*}=\max \left\{r \in \mathbb{N}: \rho\left(\boldsymbol{l}_{i}^{*}, \boldsymbol{l}_{j}^{*}\right) \geqslant \epsilon, \forall 1 \leqslant i<j \leq r\right\} .
$$

We choose $\epsilon=0.001$ in our experiments. 


\section{Numerical experiments}

In our experiments, we use the $d$-dimensional Sobol' point set as the input $\mathcal{P}=\left\{\boldsymbol{u}_{i}, i=1, \ldots, N\right\}$ for QMC. In practice, it is very hard to quantify the error of deterministic QMC quadratures. We thus use randomized QMC (RQMC) instead; see L'Ecuyer and Lemieux (2002) for a comprehensive overview of RQMC. Specifically, we obtain $M$ independent randomized versions of $\mathcal{P}$ by Owen's scrambling method (see Owen, 1995), i.e., $\mathcal{P}_{j}=\left\{\boldsymbol{u}_{i}^{(j)}, i=1, \ldots, N\right\}$ for $j=1, \ldots, M$. Correspondingly, an estimate of the option price for the $j$ th independent batch is given by $Q_{N}\left(H, \mathcal{P}_{j}, \boldsymbol{A}\right)$. Note that the randomized QMC points preserve the good quality of lowdiscrepancy points. Moreover, each element $\boldsymbol{u}_{i}^{(j)}$ of $\mathcal{P}_{j}$ has the uniform distribution in $[0,1]^{d}$, implying that $Q_{N}\left(H, \mathcal{P}_{j}, \boldsymbol{A}\right)$ is unbiased. The final estimate is the average of the $M$ independent estimates, i.e., $\bar{Q}_{N, M}(H, \boldsymbol{A})=$ $\frac{1}{M} \sum_{j=1}^{M} Q_{N}\left(H, \mathcal{P}_{j}, \boldsymbol{A}\right)$. The corresponding sample variance, which is an unbiased estimate of the variance of $\bar{Q}_{N, M}(H, \boldsymbol{A})$, is given by

$$
\hat{\sigma}_{\mathrm{QMC}}^{2}:=\frac{1}{M(M-1)} \sum_{j=1}^{M}\left(Q_{N}\left(H, \mathcal{P}_{j}, \boldsymbol{A}\right)-\bar{Q}_{N, M}(H, \boldsymbol{A})\right)^{2} .
$$

To demonstrate the efficiency of the auto-realignment method, variance reduction factors (VRFs) of using various PGMs in QMC with respect to the crude MC method are assessed and compared. The VRF measures the efficiency gain of QMC estimators as in many related literatures (see Dingeç and Hörmann, 2012). The VRF is defined by

$$
\mathrm{VRF}:=\frac{\hat{\sigma}_{\mathrm{MC}}^{2}}{\hat{\sigma}_{\mathrm{QMC}}^{2}},
$$

where $\hat{\sigma}_{\mathrm{MC}}^{2}$ denotes the sample variance of the crude MC and $\hat{\sigma}_{\mathrm{QMC}}^{2}$ denotes the sample variance (15) for QMC combined with certain PGM. Obviously, large VRF guarantees good performance of the PGM used in QMC. All the VRFs in our examples are based on 500 independent batches of simulation and each batch contains $2^{14}$ samples, i.e., $M=500$ and $N=2^{14}$. All experiments are conducted using MATLAB running on a $\mathrm{PC}$ with $3.6 \mathrm{GHz}$ Intel Core i7-4790 CPU and 16 GB RAM.

We compare six QMC-based schemes with the crude MC method (using the STD construction for generating the BM). The six QMC-based schemes 
are divided into two groups. The first group consists of Schemes 1-2 which use STD and PCA constructions to generate the BM. The second group consists of Schemes 3-6, which are problem-dependent PGMs.

(1) Standard QMC - STD,

(2) Principal Components Analysis - PCA,

(3) Orthogonal Transformation - OT,

(4) The crude QR method - CQR,

(5) The auto-realignment method with one cluster - AQR(1),

(6) The auto-realignment method with $r^{*}$ clusters - AQR* .

All the PGMs of the second group take the $\boldsymbol{A}^{\mathrm{STD}}$ as the initial generating matrix. The CQR method using normalized gradient vectors calculated on the following special points,

$$
\varepsilon_{i}=(\underbrace{1, \ldots, 1}_{i-1}, \underbrace{0, \ldots, 0}_{d-i+1}), i=1, \ldots, d .
$$

Then normalized gradient vectors $\boldsymbol{l}_{i}$ can be calculated on these points. Let the weight matrix $\boldsymbol{L}=\left(\boldsymbol{l}_{1}, \boldsymbol{l}_{2}, \ldots, \boldsymbol{l}_{d}\right) \in \mathbb{R}^{d \times d}$ and take a $\mathrm{QR}$ decomposition of $\boldsymbol{L}$ such that $\boldsymbol{L}=\boldsymbol{Q R}$. We specify the orthogonal matrix $\boldsymbol{U}$ of the CQR method by setting $\boldsymbol{U}=\boldsymbol{Q}$. This PGM is proposed for the comparison with the $\mathrm{AQR}^{*}$ method to emphasize the importance of finding representative normal vectors as columns of the weight matrix.

\subsection{Single asset case}

\subsubsection{Digital Asian option}

We first consider digital options on a single asset. The discounted payoff of a digital Asian option is given by

$$
H(\boldsymbol{x})=e^{-r T} \mathbf{1}_{\left\{S_{A}-K>0\right\}}(\boldsymbol{x}),
$$

where $S_{A}=\frac{1}{d} \sum_{j=1}^{d} S\left(t_{j}\right)$. Under the STD construction, the payoff function can be expressed in terms of $\boldsymbol{z}$

$$
h(\boldsymbol{z}):=H\left(\boldsymbol{A}^{\mathrm{STD}} \boldsymbol{z}\right)=e^{-r T} \mathbf{1}_{\{f(\boldsymbol{z})>0\}}(\boldsymbol{z}),
$$

where $\Sigma=\{\boldsymbol{z} \mid f(\boldsymbol{z})=0\}$ is the original discontinuity surface. Under the risk-neutral valuation principle, the price of the digital Asian option is given by $\mathbb{E}[h(\boldsymbol{z})]$. 
In Table 1, we present the VRFs of various QMC-based PGMs with respect to the crude $\mathrm{MC}$. In the simulation, the parameters are chosen as $S(0)=100, \sigma=0.2, r=0.1, T=1, d \in\{32,64,128\}$ and $K \in$ $\{90,100,110,120\}$. We have the following observations.

- The VRFs of QMC-based estimates are affected significantly by PGMs. The proposed $\mathrm{AQR}^{*}$ method is the most superior in terms of VRFs, irrespective of the nominal dimension and the strike price. The VRFs of using the $\mathrm{AQR}^{*}$ method in $\mathrm{QMC}$ can be at least double than those of using the other PGMs.

- The VRFs of the QMC-based STD method are much lower than those of using the other QMC-based PGMs. The VRFs of the PCA method seem to be invariant to the nominal dimension. The PCA method only provides moderate advantage over the STD method since it does not tackle the discontinuity structure of the integrand. This implies that the jump structures of the integrand have a great impact on the performance of QMC methods.

- The OT method works the best when the strike price $K=100$, and its performance gets worse when the digital Asian option is deep outthe-money or deep in-the-money. The reason is that the weight matrix chosen by the OT method is independent of the strike price $K$. So when the strike price $K$ is much larger or smaller than 100, the linear structure used by the OT method does not approximate the discontinuity structure well. The performance of the AQR(1) method and the OT method are nearly the same when the strike price $K$ is around 100, while the AQR(1) method exhibits obvious advantage over the OT method when the strike price $K=120$. This implies that the typical normal vector $\boldsymbol{l}_{1}^{*}$ chosen by the auto-realignment method approximates the discontinuity surface better than the OT method when the digital Asian option is deep out-the-money or deep in-the-money.

- The efficiency gain of the AQR* method is significantly larger than the AQR(1) method, which implies that the discontinuities of the payoff function cannot be approximated by just one structure. Thus the AQR* method which chooses $r^{*}$ most representative normal vectors $\boldsymbol{l}_{1}^{*}, \ldots, \boldsymbol{l}_{r^{*}}^{*}$ is much better than just applying single structure. 
Table 1: The variance reduction factors for the digital Asian option (16).

\begin{tabular}{|c|c|c|c|c|c|c|c|c|}
\hline \multirow{2}{*}{$d$} & \multirow{2}{*}{ K } & $\mathrm{MC}$ & \multicolumn{6}{|c|}{ Sobol' } \\
\hline & & STD & STD & $\mathrm{PCA}$ & $\mathrm{OT}$ & CQR & $\mathrm{AQR}(1)$ & $\mathrm{AQR}^{*}$ \\
\hline \multirow{4}{*}{32} & 90 & 1 & 3 & 38 & 94 & 95 & 108 & 214 \\
\hline & 100 & 1 & 4 & 57 & 161 & 153 & 147 & 363 \\
\hline & 110 & 1 & 4 & 63 & 126 & 153 & 161 & 309 \\
\hline & 120 & 1 & 3 & 48 & 69 & 91 & 108 & 236 \\
\hline \multirow{4}{*}{64} & 90 & 1 & 3 & 41 & 70 & 89 & 86 & 214 \\
\hline & 100 & 1 & 3 & 58 & 102 & 141 & 106 & 349 \\
\hline & 110 & 1 & 3 & 60 & 85 & 123 & 107 & 311 \\
\hline & 120 & 1 & 3 & 48 & 59 & 79 & 89 & 219 \\
\hline \multirow{4}{*}{128} & 90 & 1 & 2 & 39 & 72 & 67 & 82 & 206 \\
\hline & 100 & 1 & 3 & 61 & 98 & 102 & 98 & 314 \\
\hline & 110 & 1 & 3 & 60 & 95 & 102 & 103 & 315 \\
\hline & 120 & 1 & 3 & 48 & 50 & 71 & 84 & 245 \\
\hline
\end{tabular}

Note: The parameters are $S(0)=100, \sigma=0.2, r=0.1, T=1$. These values are calculated based on 500 independent batches of simulation and each batch contains $2^{14}$ samples.

- The comparison of results of the CQR method and the AQR* method confirms that the procedure of finding several representative normal vectors of the discontinuity surface is indispensable. If we use the normalized gradient vectors calculated on $d$ arbitrarily chosen points to approximate the discontinuity surface, the performance of the CQR method is not very well compared with the AQR* method and even worse than $\mathrm{AQR}(1)$ in some cases.

Next we turn to the convergence graphs in Figure 1 for the digital Asian option. The parameters are given as $d \in\{64,128\}$ and $K=100$. In these graphs, the horizontal axis gives the number of low discrepancy points $N$ (which ranges from $2^{0}$ to $2^{20}$ ) plotted on a log scale. The vertical axis gives the mean squared error (MSE) obtained from 500 independent simulations also plotted on a log scale. The RQMC method yields an unbiased estimator. Thus we use the sample variance as the estimate of the MSE. Figure 1 shows 

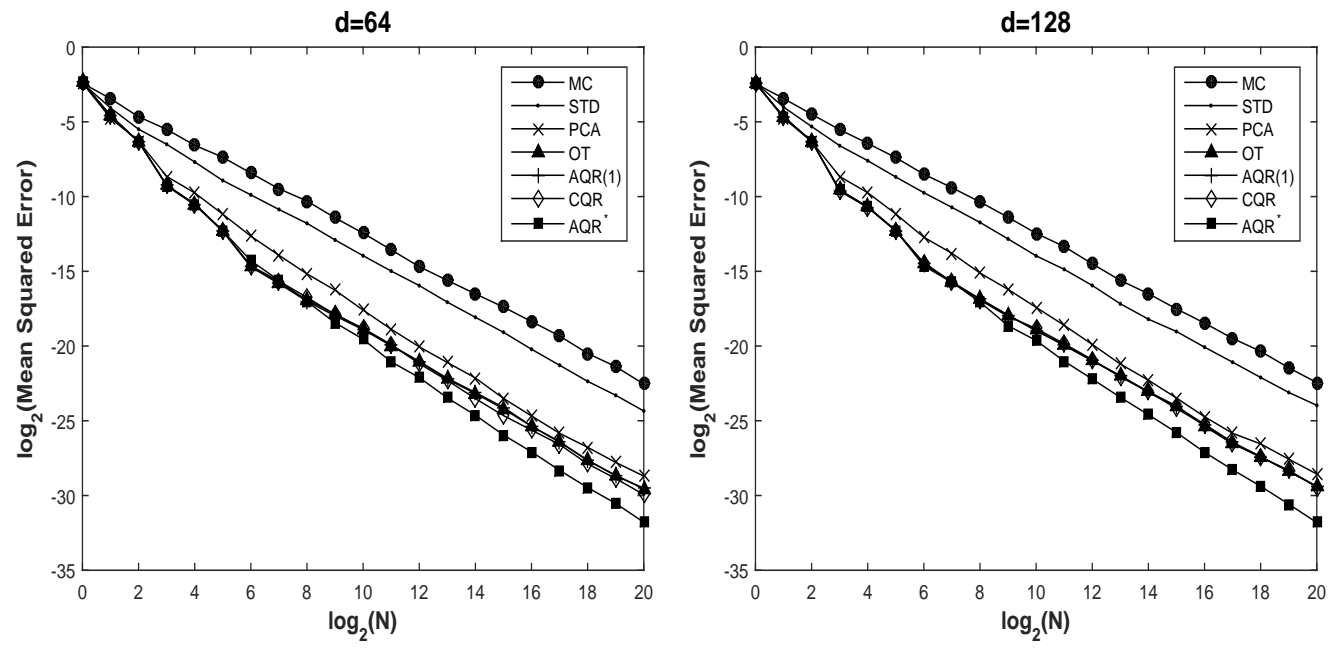

Figure 1: MSE versus $N$ (log-log plot) for digital Asian option $(d \in\{64,128\}, K=100)$.

that the $\mathrm{AQR}^{*}$ method has the least MSE and exhibits the fastest convergence rate among other PGMs. Thus the superiority of the $\mathrm{AQR}^{*}$ method over the other PGMs is confirmed.

\subsubsection{Digital Asian barrier option}

Consider the following digital Asian barrier option with a payoff as

$$
H(\boldsymbol{x})=e^{-r T} \mathbf{1}_{\left\{S_{A}-K>0\right\}}(\boldsymbol{x}) \mathbf{1}_{\left\{S_{A}-B<0\right\}}(\boldsymbol{x}),
$$

where $B>K$ is the barrier. Under STD construction, the payoff function can be expressed in terms of $\boldsymbol{z}$. Let $g(\boldsymbol{x})=\min \left(S_{A}-K, B-S_{A}\right)$ and $f(\boldsymbol{z}):=g\left(\boldsymbol{A}^{\mathrm{STD}} \boldsymbol{z}\right)$. Note that $f(\boldsymbol{z})$ is a continuous and almost everywhere differentiable function. The payoff function can be expressed as

$$
h(\boldsymbol{z}):=e^{-r T} \mathbf{1}_{\{f(\boldsymbol{z})>0\}}(\boldsymbol{z}) .
$$

In Table 2, we present the VRFs of various QMC-based PGMs with respect to the crude MC. We choose the parameters as $S(0)=100, \sigma=0.2$, $r=0.1, T=1, K=100, B \in\{120,130,140\}, d \in\{32,64,128\}$. We draw the following remarks based on the simulation results.

- The $\mathrm{AQR}^{*}$ method is the most superior in terms of VRFs, irrespective of the nominal dimension and the barrier. 
Table 2: The variance reduction factors for the digital Asian barrier option (17).

\begin{tabular}{|c|c|c|c|c|c|c|c|c|}
\hline \multirow{2}{*}{$d$} & \multirow{2}{*}{$(\mathrm{K}, \mathrm{B})$} & $\mathrm{MC}$ & \multicolumn{6}{|c|}{ Sobol' } \\
\hline & & STD & STD & $\mathrm{PCA}$ & $\mathrm{OT}$ & CQR & $\mathrm{AQR}(1)$ & $\mathrm{AQR}^{*}$ \\
\hline \multirow{3}{*}{32} & $(100,120)$ & 1 & 2 & 35 & 72 & 87 & 77 & 179 \\
\hline & $(100,130)$ & 1 & 3 & 49 & 87 & 104 & 95 & 232 \\
\hline & $(100,140)$ & 1 & 4 & 52 & 110 & 115 & 110 & 235 \\
\hline \multirow{3}{*}{64} & $(100,120)$ & 1 & 2 & 45 & 67 & 83 & 70 & 207 \\
\hline & $(100,130)$ & 1 & 3 & 44 & 67 & 97 & 68 & 231 \\
\hline & $(100,140)$ & 1 & 3 & 52 & 90 & 117 & 94 & 262 \\
\hline \multirow{3}{*}{128} & $(100,120)$ & 1 & 2 & 40 & 51 & 67 & 61 & 196 \\
\hline & $(100,130)$ & 1 & 3 & 56 & 84 & 94 & 92 & 274 \\
\hline & $(100,140)$ & 1 & 3 & 58 & 90 & 99 & 93 & 273 \\
\hline
\end{tabular}

Note: The parameters are $S(0)=100, \sigma=0.2, r=0.1, T=1$.

- The OT method and the crude QR method are affected by the nominal dimension of the problem. These two methods perform better in low-dimensional cases than in high-dimensional cases. When the strike price $K$ and the barrier $B$ are closer, the efficiency of these two methods gets worse. The performance of the AQR(1) method and the OT method are nearly the same in this case.

- The efficiency gain of the $\mathrm{AQR}^{*}$ method is significantly larger than the AQR(1) method. This confirms that the AQR* method which chooses $r^{*}$ representative normal vectors is much better than the $\mathrm{AQR}(1)$ method which chooses only one structure.

The convergence graphs of MSE for the digital Asian barrier option is presented in Figure 2. The parameters are given as $d \in\{64,128\}$ and $B=$ 130. The results are also very encouraging. The AQR* method has the least MSE and exhibits the fastest convergence rate among various PGMs. The comparison confirms the superiority of the $\mathrm{AQR}^{*}$ method over other PGMs.

\subsection{Multi-asset case}

In this subsection we consider the case of digital option based on multiple underlying assets. We assume that the risky assets follow the multi- 

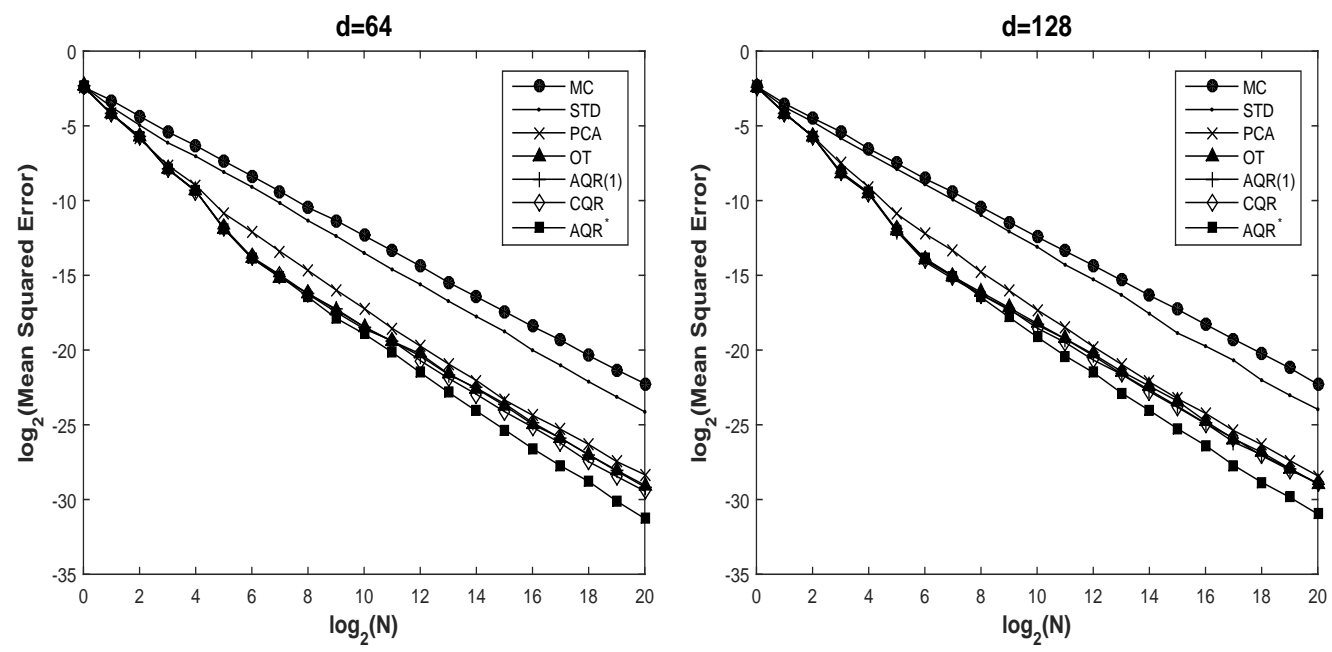

Figure 2: MSE versus $N$ (log-log plot) for digital Asian barrier option $(d \in\{64,128\}$, $B=130)$.

dimensional geometric BM. Under the risk-neutral measure, their dynamics are given by the stochastic differential equations

$$
d S_{i}(t)=r S_{i}(t) d t+\sigma_{i} S_{i}(t) d B_{i}(t), i=1, \ldots, d,
$$

where $S_{i}(t)$ denotes the $i$ th asset price at time $t, r$ is the interest rate, $\sigma_{i}$ is the volatility for the $i$ th asset, and $\boldsymbol{B}(t)=\left(B_{1}(t), \ldots, B_{d}(t)\right)^{T}$ is a $d$ dimensional BM, where each $B_{i}(t)$ is a standard one-dimensional BM and the instantaneous correlation of $B_{i}(t)$ and $B_{j}(t)$ is $C_{i j}$,

$$
\operatorname{Cov}\left(B_{j}(t), B_{j}(t)\right)=C_{i j} t
$$

where the matrix $\boldsymbol{C}=\left(C_{i j}\right)_{d \times d}$ is positive definite.

Suppose that the $d$-dimensional vector $\mathbf{S}:=\left(S_{1}(T), S_{2}(T), \ldots, S_{d}(T)\right)^{T}$ represents the price of underlying assets at time $T$ and the $j$ th asset price at time $T$ is given by

$$
S_{j}(T)=S_{j}(0) \exp \left\{\left(r-\sigma_{j}^{2} / 2\right) T+\sigma_{j} B_{j}(T)\right\}, j=1, \ldots, d .
$$

For simplicity we assume the initial value $S_{i}(0)=S(0)$ and the volatility $\sigma_{i}=\sigma$ for $i=1, \ldots, d$. Let $\boldsymbol{x}=\left(x_{1}, \ldots, x_{d}\right)^{T}:=\left(B_{1}(T), \ldots, B_{d}(T)\right)^{T}$, then $\boldsymbol{x} \sim N(\mathbf{0}, \boldsymbol{C})$. Consider a digital Asian option based on the weighted 
arithmetic average of the multi-asset prices. The discounted payoff function is given as following,

$$
H(\boldsymbol{x})=e^{-r T} \mathbf{1}_{\left\{S_{A}(\boldsymbol{\beta})-K>0\right\}}(\boldsymbol{x}),
$$

where $S_{A}(\boldsymbol{\beta})=\sum_{j=1}^{d} \beta_{j} S_{j}(T)$ and $\boldsymbol{\beta}=\left(\beta_{1}, \ldots, \beta_{d}\right)^{T}$ is a vector satisfying $\sum_{j=1}^{d} \beta_{j}=1$. By taking Cholesky decomposition of the covariance matrix $\boldsymbol{C}$, we get the STD generating matrix $\boldsymbol{A}^{\mathrm{STD}}$ of the BM. So $\boldsymbol{x}$ can be represented as $\boldsymbol{x}=\boldsymbol{A}^{\mathrm{STD}} \boldsymbol{z}, \boldsymbol{z} \sim N\left(\mathbf{0}, \boldsymbol{I}_{d}\right)$. Let $h(\boldsymbol{z}):=H\left(\boldsymbol{A}^{\mathrm{STD}} \boldsymbol{z}\right)$ expressed in terms of $\boldsymbol{z}$, i.e., $h(\boldsymbol{z}):=e^{-r T} \mathbf{1}_{\{f(\boldsymbol{z})>0\}}(\boldsymbol{z})$, where $\Sigma=\{\boldsymbol{z} \mid f(\boldsymbol{z})=0\}$ is the discontinuity surface.

In Table 3, we present the VRFs of various QMC-based PGMs with respect to the crude MC. We set the parameters as $S(0)=100, \sigma=0.2$, $r=0.1, T=1, d \in\{50,100\}$, and $K \in\{90,100,110,120\}$. In our experiments, we study two kinds of covariance matrices $\boldsymbol{C}$, where $\boldsymbol{C}_{1}=\left(C_{i j}\right)_{d \times d}$ in which $C_{i j}=T(d-|i-j|) / d$ and $C_{2}=\left(C_{i j}\right)_{d \times d}$ in which $C_{i j}=T \lambda^{|i-j|}$ with $\lambda=0.98$. The weight vector $\boldsymbol{\beta}$ is chosen from one of the following, $\boldsymbol{\beta}_{1}=(1 / d, \ldots, 1 / d)^{T}$ and $\boldsymbol{\beta}_{2}=c(|\cos (2 \pi / d)|, \ldots,|\cos (2 d \pi / d)|)^{T}$, where $c$ is a positive number such that $\sum_{j=1}^{d} \beta_{j}=1$. We have the following observations.

- The $\mathrm{AQR}^{*}$ method in QMC performs consistently better than STD, PCA, OT, CQR and AQR(1), regardless of the nominal dimension, the strike price, the choice of covariance matrix $\boldsymbol{C}$ and the vector $\boldsymbol{\beta}$.

- The VRFs of the PCA method and the crude QR method seem to perform well when the weight vector $\boldsymbol{\beta}=\boldsymbol{\beta}_{1}$. But for the weight vector $\boldsymbol{\beta}=\boldsymbol{\beta}_{2}$, the efficiency of the PCA method and the CQR method decreases. This implies that the PCA method and the CQR method may not be a stable good PGM. The CQR which chooses $d$ arbitrary structures may be inappropriate.

- The performance of the AQR(1) method and the OT method are nearly the same observed from Table 3. The comparison of the AQR* method and the OT method implies that it is insufficient to use only one normal vector to handle the discontinuities. The normal vectors on the discontinuity surface of the payoff function may be distributed more dispersedly than in the single asset case, which may be the reason of the inefficiency of the OT method and the AQR(1) method. 
Table 3: The variance reduction factors for the digital multi-asset option (18).

\begin{tabular}{|c|c|c|c|c|c|c|c|c|c|}
\hline \multirow{2}{*}{$(\boldsymbol{C}, \boldsymbol{\beta})$} & \multirow{2}{*}{$\mathrm{d}$} & \multirow{2}{*}{ K } & $\mathrm{MC}$ & \multicolumn{6}{|c|}{ Sobol' } \\
\hline & & & STD & STD & PCA & $\mathrm{OT}$ & CQR & $\operatorname{AQR}(1)$ & $\mathrm{AQR}^{*}$ \\
\hline \multirow{8}{*}{$\left(\boldsymbol{C}_{1}, \boldsymbol{\beta}_{1}\right)$} & \multirow{4}{*}{50} & 90 & 1 & 3 & 70 & 43 & 103 & 45 & 124 \\
\hline & & 100 & 1 & 4 & 112 & 52 & 134 & 51 & 178 \\
\hline & & 110 & 1 & 4 & 113 & 53 & 140 & 53 & 196 \\
\hline & & 120 & 1 & 3 & 100 & 45 & 127 & 45 & 164 \\
\hline & \multirow{4}{*}{100} & 90 & 1 & 3 & 75 & 28 & 97 & 29 & 136 \\
\hline & & 100 & 1 & 3 & 110 & 31 & 120 & 31 & 175 \\
\hline & & 110 & 1 & 4 & 112 & 31 & 109 & 31 & 181 \\
\hline & & 120 & 1 & 3 & 101 & 32 & 110 & 33 & 172 \\
\hline \multirow{8}{*}{$\left(\boldsymbol{C}_{1}, \boldsymbol{\beta}_{2}\right)$} & \multirow{4}{*}{50} & 90 & 1 & 3 & 45 & 38 & 47 & 38 & 115 \\
\hline & & 100 & 1 & 3 & 61 & 54 & 57 & 56 & 178 \\
\hline & & 110 & 1 & 4 & 59 & 47 & 57 & 46 & 154 \\
\hline & & 120 & 1 & 3 & 74 & 52 & 61 & 52 & 164 \\
\hline & \multirow{4}{*}{100} & 90 & 1 & 3 & 43 & 28 & 67 & 30 & 126 \\
\hline & & 100 & 1 & 3 & 59 & 29 & 85 & 30 & 169 \\
\hline & & 110 & 1 & 4 & 68 & 33 & 98 & 33 & 188 \\
\hline & & 120 & 1 & 3 & 70 & 40 & 95 & 39 & 176 \\
\hline \multirow{8}{*}{$\left(\boldsymbol{C}_{2}, \boldsymbol{\beta}_{1}\right)$} & \multirow{4}{*}{50} & 90 & 1 & 3 & 92 & 58 & 102 & 59 & 153 \\
\hline & & 100 & 1 & 4 & 120 & 73 & 119 & 76 & 186 \\
\hline & & 110 & 1 & 5 & 131 & 84 & 132 & 83 & 224 \\
\hline & & 120 & 1 & 4 & 141 & 81 & 145 & 80 & 218 \\
\hline & \multirow{4}{*}{100} & 90 & 1 & 3 & 49 & 23 & 47 & 24 & 83 \\
\hline & & 100 & 1 & 3 & 71 & 27 & 54 & 28 & 102 \\
\hline & & 110 & 1 & 3 & 70 & 27 & 69 & 27 & 104 \\
\hline & & 120 & 1 & 3 & 62 & 28 & 59 & 27 & 105 \\
\hline \multirow{8}{*}{$\left(\boldsymbol{C}_{2}, \boldsymbol{\beta}_{2}\right)$} & \multirow{4}{*}{50} & 90 & 1 & 3 & 61 & 55 & 99 & 58 & 154 \\
\hline & & 100 & 1 & 4 & 76 & 75 & 113 & 74 & 196 \\
\hline & & 110 & 1 & 5 & 92 & 86 & 112 & 88 & 223 \\
\hline & & 120 & 1 & 4 & 86 & 80 & 105 & 82 & 184 \\
\hline & \multirow{4}{*}{100} & 90 & 1 & 3 & 36 & 27 & 48 & 29 & 104 \\
\hline & & 100 & 1 & 3 & 49 & 30 & 54 & 31 & 115 \\
\hline & & 110 & 1 & 4 & 52 & 30 & 59 & 30 & 134 \\
\hline & & 120 & 1 & 3 & 47 & 27 & 58 & 26 & 105 \\
\hline
\end{tabular}

Note: The parameters are $S(0)=100, \sigma=0.2, r=0.1, T=1$. 

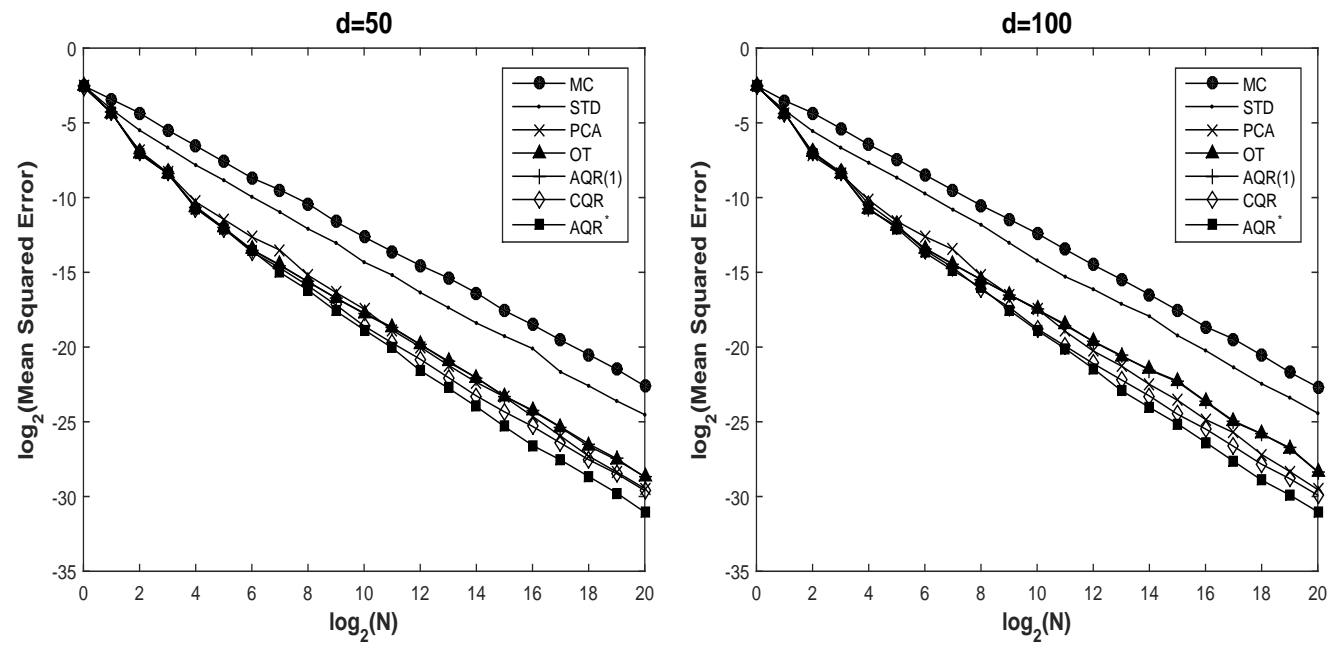

Figure 3: MSE versus $N$ (log-log plot) for digital multi-asset option $(18)(d \in\{50,100\})$.

Thus choosing enough representative normal vectors on the discontinuity surface is indispensable when we tackle the complex discontinuity structures.

Figure 3 presents the convergence graphs of digital multi-asset option for $d \in\{50,100\}$. We choose $K=100, \boldsymbol{C}=\boldsymbol{C}_{1}$ and $\boldsymbol{\beta}=\boldsymbol{\beta}_{2}$. Obviously, the $\mathrm{AQR}^{*}$ method has the least MSE and exhibits the fastest convergence rate among all PGMs. The comparison once more confirms the superiority of the $\mathrm{AQR}^{*}$ method over the other PGMs.

\section{Conclusions}

Many financial derivatives involve jump structures in their payoff functions. These structures may deteriorate the efficiency of the QMC method significantly. Although the OT method can recover the superiority of the QMC method for problems involving simple discontinuity structures, but for those payoffs involving complex discontinuities, they cannot be applied directly.

The contribution of this paper is twofold. Firstly, we use the machine learning technique to learn the information of the complex discontinuities more thoroughly in an automatic way. Learning the nature of the discontinuities completely is the key step to design a problem-dependent PGM. 
The second contribution of this paper is to propose a new PGM called the auto-realignment method to handle complex discontinuities. The proposed method finds several representative normal vectors of the discontinuity surface by using the $k$-means clustering algorithm. After finding the appropriate weight matrix, we use the $\mathrm{QR}$ method to realign the discontinuity surface such that it is parallel to as many coordinate axes as possible. Thus the discontinuity structure of the transformed function will be much more QMCfriendly. As illustrated in the experiments, the proposed PGM is effective and robust.

The idea of machine learning plays an important role in designing the new PGM. We expect to find a better PGM via more efficient and accurate machine learning techniques. As future research, it is worth developing similar methods for pricing options and estimating Greeks under Lévy processes.

\section{Acknowledgments}

The authors thank the support from the National Science Foundation of China under grant 71471100.

\section{References}

Acworth, P., Broadie, M., Glasserman, P., 1998. A comparison of some Monte Carlo and quasi Monte Carlo techniques for option pricing. In: Monte Carlo and Quasi-Monte Carlo Methods 1996. Springer, pp. 1-18.

Caflisch, R. E., Morokoff, W. J., Owen, A. B., 1997. Valuation of mortgage backed securities using Brownian bridges to reduce effective dimension. J. Comput. Finance 1 (1), 27-46.

Dingeç, K. D., Hörmann, W., 2012. A general control variate method for option pricing under Lévy processes. European J. Oper. Res. 221 (2), 368377.

Glasserman, P., 2004. Monte Carlo Methods in Financial Engineering. Vol. 53. Springer.

Golub, G. H., van Loan, C. F., 2013. Matrix Computations, 4th Edition. Vol. 3. Jphn Hopkins University Press, Baltimore, MD. 
Hartigan, J. A. ., Wong, M. A., 1979. A k-means clustering algorithm. J. R. Stat. Soc. Ser. C. Appl. Stat. 28, 100-108.

He, Z., Wang, X., 2014. Good path generation methods in quasi-Monte Carlo for pricing financial derivatives. SIAM J. Sci. Comput. 36 (2), B171-B197.

Imai, J., Tan, K. S., 2006. A general dimension reduction technique for derivative pricing. J. Comput. Finance 10 (2), 129-155.

Jin, X., Li, X., Tan, H. H., Wu, Z., 2013. A computationally efficient statespace partitioning approach to pricing high-dimensional American options via dimension reduction. European J. Oper. Res. 231 (2), 362-370.

Joy, C., Boyle, P., Tan, K. S., 1996. Quasi-Monte Carlo methods in numerical finance. Management Sci. 42 (6), 926-938.

L'Ecuyer, P., Lemieux, C., 2002. Recent advances in randomized quasi-Monte Carlo methods. In: M. Dror, P. L., Szidarovszki, F. (Eds.), Modeling Uncertainty: An Examination of Stochastic Theory, Methods, and Applications. Kluwer Academic, Boston, pp. 419-474.

Moskowitz, B., Caflisch, R. E., 1996. Smoothness and dimension reduction in quasi-Monte Carlo methods. Math. Comput. Modelling 23 (8), 37-54.

Niederreiter, H., 1992. Random Number Generation and Quasi-Monte Carlo Methods. SIAM, Philadelphia.

Owen, A. B., 1995. Randomly permuted $(t, m, s)$-nets and $(t, s)$-sequences. In: Niederreiter, H., Shiue, P. J.-S. (Eds.), Monte Carlo and Quasi-Monte Carlo Methods in Scientific Computing. Springer, pp. 299-317.

Paskov, S. H., Traub, J. F., 1995. Faster valuation of financial derivatives. J. Portfolio Management 22 (1), 113-123.

Wang, X., 2016. Handling discontinuities in financial enginnering: good path simulation and smoothing. Oper. Res. To appear.

Wang, X., Fang, K.-T., 2003. The effective dimension and quasi-Monte Carlo integration. J. Complexity 19 (2), 101-124.

Wang, X., Tan, K., 2013. Pricing and hedging with discontinuous functions: quasi-Monte Carlo methods and dimension reduction. Management Sci. 59 (2), 376-389. 